Planetary Systems in the Universe - Observation, Formation and Evolution

Proceedings IAU Symposium No. 202, (c)2004 IAU

Alan Penny, Pawel Artymowicz, Anne-Marie Lagrange, $\&$ Sara Russell, eds.

\title{
The Role of Clouds in Brown Dwarf and Extrasolar Giant Planet Atmospheres
}

\author{
Mark S. Marley \\ NASA Ames Research Center; Mail Stop 245-3; Moffett Field, CA and \\ New Mexico State University; Department of Astronomy; Las Cruces, \\ NM 88003, USA \\ Andrew S. Ackerman \\ NASA Ames Research Center; Mail Stop 245-4; Moffett Field, \\ $C A$ 94035, USA
}

\begin{abstract}
Clouds and hazes are important throughout our solar system and in the atmospheres of brown dwarfs and extrasolar giant planets. Among the brown dwarfs, clouds control the colors and spectra of the L-dwarfs; the disappearance of clouds helps herald the arrival of the T-dwarfs. The structure and composition of clouds will be among the first remote-sensing results from the direct detection of extrasolar giant planets.
\end{abstract}

\section{Introduction}

Even before the first discovery of brown dwarfs and extrasolar giant planets (EGPs) it had been apparent that a detailed appreciation of cloud physics would be required to understand the atmospheres of these objects (e.g. Lunine et al. 1989). Depending on the atmospheric effective temperature, $\mathrm{Fe}, \mathrm{Mg}_{2} \mathrm{SiO}_{4}$, $\mathrm{MgSiO}_{3}, \mathrm{H}_{2} \mathrm{O}$, and $\mathrm{NH}_{3}$ among others may condense in substellar atmospheres. Since every atmosphere in the solar system is influenced by clouds, dust, or hazes, the need to follow the fate of condensates in brown dwarf and EGP atmospheres is self-evident. What has become clearer over the past five years is that details such as the vertical structure and particle sizes in clouds play a decisive role in controlling the thermal structure and emergent spectra from these atmospheres. Indeed the available data are already sufficient to help us choose among competing models.

In this contribution we will briefly summarize some of the roles clouds play in a few solar system atmospheres to illustrate what might be expected of brown dwarf and extrasolar giant planet atmospheres. Then we will summarize a new cloud model developed to study these effects, present some model results, and compare them to data. Since brown dwarfs have similar compositions and effective temperatures to EGPs and a rich dataset already exists, we focus on the lessons learned from the L- and T-dwarfs. We then briefly review the importance of clouds to EGP atmospheres and future observations. 


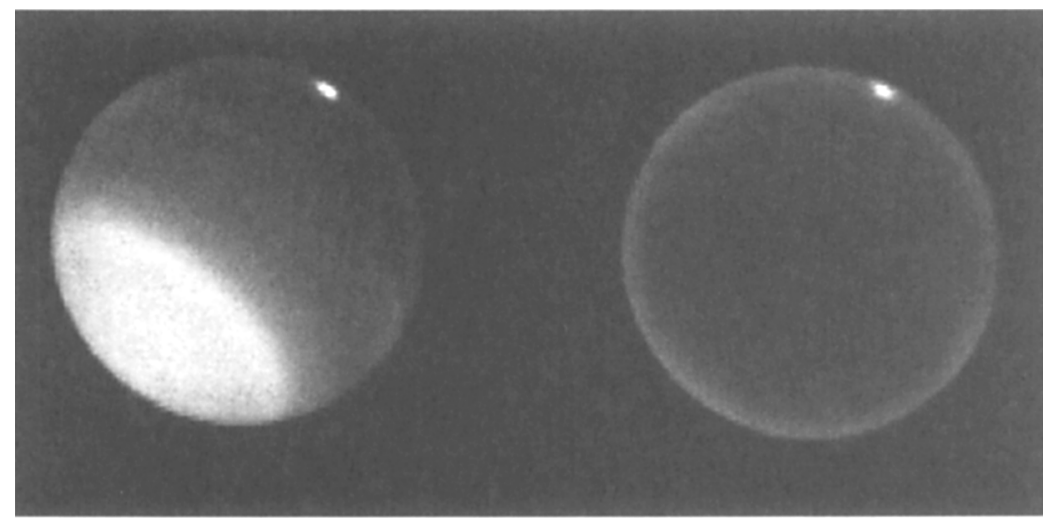

Figure 1. Near consecutive HST images of Uranus taken through different filters. The filter employed for the left hand image probes a broad spectral range from 0.85 to $1 \mu \mathrm{m}$ while the right hand image is taken through a narrow filter sensitive to the $0.89 \mu \mathrm{m} \mathrm{CH}$ absorption band. The relative visibility of various cloud features between the two images is a measure of the cloud height as the incident photon penetration depth is modulated by methane absorption. Images courtesy H. Hammel and K. Rages.

\section{Clouds in the Solar System}

Clouds dramatically alter the appearance, thermal structure, and even evolution of planets. Venus glistens white in the morning and evening skies because sunlight reflects off of its bright cloud tops. If there were no condensates in Venus' atmosphere the planet would take on a bluish hue from Rayleigh scattered sunlight. Mars' atmosphere is warmer than it would otherwise be thanks to absorption of incident solar radiation by atmospheric dust (Pollack et al. 1979). The effectiveness of the $\mathrm{CO}_{2}$ greenhouse during Mars's putative warm and wet early history is tied to poorly understood details of its cloud physics and radiative transfer (Mischna, et al. 2000). Indeed the future climate of Earth in a fossil-fuel-fired greenhouse may hinge on the role water clouds will play in altering Earth's albedo and scattering or absorbing thermal radiation.

The appearance of the Jovian planets is controlled by the extensive cloud decks covering their disks. On Jupiter and Saturn thick $\mathrm{NH}_{3}$ clouds, contaminated by an unknown additional absorber, reflect about $35 \%$ of incident radiation back to space. $\mathrm{CH}_{4}$ and $\mathrm{H}_{2} \mathrm{~S}$ clouds play a similar role at Uranus and Neptune.

The vertical structure of the jovian cloud layers was deduced by variation of their reflected spectra inside and outside of molecular absorption bands. Figure 1 illustrates this process. In the left hand image incident sunlight penetrates relatively deeply into the atmosphere and is scattered principally by a cloud deck over the south pole and a bright cloud near the northern mid-latitude limb. The relative heights of these two features cannot be discerned from this single image. The right hand image, however, was taken in the strong $0.89-\mu \mathrm{m}$ methane absorption band. Here the south polar cloud is invisible since incident 
sunlight is absorbed by $\mathrm{CH}_{4}$ gas above the cloud before it can scatter. We conclude that the bright northern cloud lies higher in the atmosphere since it is still visible in this image. The application of this technique to spectra and images of the giant planets has yielded virtually all the information we have about the vertical structure of these atmospheres (e.g. West, Strobel, \& Tomasko 1986; Baines \& Hammel 1994; Baines et al. 1995). A similar reasoning process can be applied to brown dwarf and EGP atmospheres.

The large body of work on jovian clouds cannot be easily generalized, but two robust results are apparent. First, sedimentation of cloud droplets is important. Cloud particles condense from the atmosphere, coagulate, and fall. The fall velocity depends on the size of the drops and the upward velocity induced by convection or other motions in the atmosphere. They do not stay put. A diagnostic often retrieved from imaging or spectroscopic observations of clouds is the ratio of the cloud particle scale height to that of the gas. If condensates were distributed uniformly vertically in the atmosphere this ratio would be 1 . Instead numerous investigations have found a ratio for Jupiter's ammonia clouds of about 0.3 (Carlson, Lacis, \& Rossow 1994). The clouds are thus relatively thin in vertical extent. The importance of sedimentation is borne out even for unseen Fe clouds, for example, by Jupiter's atmospheric chemistry (Fegley \& Lodders 1994).

A second important result is that cloud particles are large, a result of coagulation processes within the atmosphere. Sizes are difficult to infer remotely and the sizes to which a given observation is sensitive depend upon the wavelength observed. Nevertheless it is clear that Jupiter's ammonia clouds include particles with radii exceeding 1 to $10 \mu \mathrm{m}$, much larger than might be expected simply by direct condensation from vapor in the presence of abundant condensation nuclei (Carlson et al. 1994; Brooke et al. 1996). Similar results are found for ammonia clouds on Saturn (Tomasko et al. 1984) and methane clouds in Uranus and Neptune (Baines et al. 1995).

These two lessons from the solar jovian atmospheres - clouds have finite vertical extents governed by sedimentation and large condensate sizes - guide us as we consider clouds in brown dwarf and extrasolar giant planet atmospheres.

\section{Evidence of Clouds in Brown Dwarf Atmospheres}

The first models of the prototypical T-dwarf Gl 229 B established that grains play a minor role, if any, in controlling the spectrum of the object. The early Gl 229 B models of Marley et al. (1996), Allard et al. (1996) and Tsuji et al. (1996) all found best fits to the observed spectrum by neglecting grain opacity. This provided strong evidence that any cloud layer was confined below the visible atmosphere. All the models, however, shared the same shortcoming of predicting infrared water bands deeper than observed. Another difficulty with the early models is that they either predicted too much flux shortwards of $1 \mu \mathrm{m}$ (Marley at al. 1996) or used unrealistic molecular opacities (Allard et al. 1996) to lower the optical flux. Griffith, Yelle, \& Marley (1999) and Tsuji, Ohnaka, \& Aoki (1999) suggested variations of particulate opacity to lower the flux, but ultimately Burrows, Marley, \& Sharp (2000) argued that broadened alkali metal 


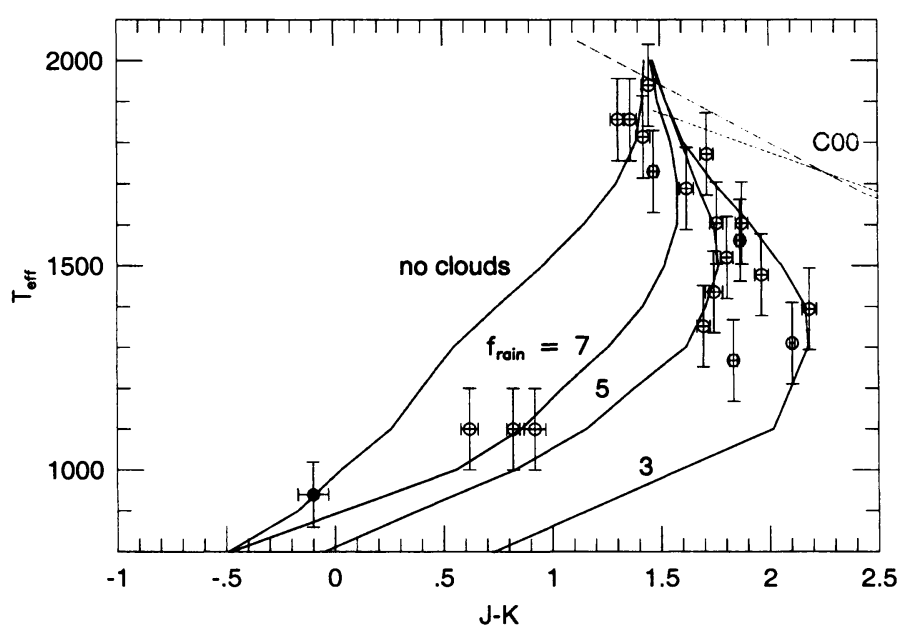

Figure 2. $\quad J-K$ color of brown dwarfs as a function of $T_{\text {eff }}$. Open data points represent L- and T-dwarf colors measured by Stephens et al. (2001) with L-dwarf temperatures estimated from fits of $K-L^{\prime}$ to models of Marley et al. (2001). Since $K-L^{\prime}$ is relatively insensitive to the presence or absence of clouds for the L-dwarfs it provides a good $T_{\text {eff }}$ scale (Marley et al. 2001). The early T-types $(0.5<J-K<1)$ are arbitrarily all assigned to $T_{\text {eff }}=1100 \mathrm{~K}$. Likewise model $T_{\text {eff }}$ s are given estimated error bars of $\pm 100 \mathrm{~K}$. The filled circle represents the position of the prototypical T-dwarf Gl 229 B (Saumon et al. 2000; Leggett et al. 1999). Four model cases are shown from the work of Marley et al. (2001): evolution with no clouds, and with clouds following the prescription of Ackerman \& Marley (2001) with $f_{\text {rain }}$ (rainfall efficiency, see text) varying from 7 (heavy rainfall) to 3 (moderate rain). Also shown are colors (C00) from models by Chabrier et al. (2000) in which there is no downward transport of condensate. The Marley et al. model lines are for objects with gravity $g=1000 \mathrm{~m} \mathrm{sec}^{-2}$, roughly appropriate for a $30 \mathrm{M}_{\mathrm{J}}$ object. There is little dependence of $J-K$ on gravity in this regime. The Chabrier et al. lines are for 30 and $60 \mathrm{MJ}$ objects.

bands were responsible for the diminution in flux, a prediction verified by Liebert et al. (2000).

The first confirmation that dust was present in the atmospheres of at least some brown dwarfs came with the discovery of the warmer L-dwarfs. These objects, unlike the methane-dominated T-dwarfs, have red colors in $J-K$ and spectra that have been best fit with dusty atmosphere models (Jones \& Tsuji 1997), although a complete analysis does not yet exist. The difficulty arose in explaining how the dusty, red L-dwarfs evolved into the clear, blue T-dwarfs (Figure 2). Models in which dust does not settle into discrete cloud layers (Chabrier et al. 2000) predict that cooling brown dwarfs would become redder in $J-K$ with falling effective temperature as more and more dust dominates the atmosphere. Since the atmosphere models employed in this work ignore the lessons learned from our jovian planets (they employ sub-micron particle sizes and do not allow the dust to settle) it is not surprising that they do not fit the data. 


\section{A New Cloud Model}

A number of models have been developed to describe the cloud formation processes in giant planet and brown dwarf atmospheres. Ackerman \& Marley (2001) describe these in some detail. In general these models suffer from a number of drawbacks which limit their utility for brown dwarf and EGP modeling. Some rely upon free parameters which are almost impossible to predict while others do not predict quantities relevant to radiative transfer of in the atmosphere. For example, the atmospheric supersaturation cannot be specified without a detailed knowledge of the number of condensation nuclei available. Ackerman \& Marley developed a new eddy sedimentation model for cloud formation in substellar atmospheres that attempts to predict cloud particle sizes and vertical extents.

Ackerman \& Marley argue that in terrestrial clouds the downward transport of large drops as rain removes substantial mass from clouds and reduces their optical depth. Yet properly modeling the condensation, coagulation, and transport of such drops requires a complex microphysical model and a concomitant abundance of free parameters. In an attempt to account for the expected effects of such microphysical processes without modeling them in detail, they introduce a new term into the equation governing the mass fraction $q_{t}$ of an atmospheric condensate at a given altitude $z$ in an atmosphere:

$$
K \frac{\partial q_{t}}{\partial z}+f_{\mathrm{rain}} w_{*} q_{c}=0
$$

Here the upward transport of the vapor and condensate is by eddy diffusion as parameterized by an eddy diffusion coefficient $K$. In equilibrium this upward transport is balanced by the downward transport of condensate $q_{c}$. The free parameter $f_{\text {rain }}$ has been introduced as the ratio of the mass-weighted droplet sedimentation velocity to $w_{*}$, the convective velocity scale. In essence $f_{\text {rain }}$ allows downward mass transport to be dominated by massive drops larger than the scale set by the local eddy updraft velocity: in other words, rain. Ackerman \& Marley (2001) treat $f_{\text {rain }}$ as an adjustable parameter and explore its consequences.

\section{Clouds and the L- to T-dwarf transition}

Given the importance of clouds to the L-dwarf spectra and the absence of significant cloud opacity in the T-dwarfs, it is clear that the departure of clouds with falling $T_{\text {eff }}$ is an important milestone in the transition from L- to T-dwarfs. Marley (2000) demonstrated that a simple cloud model in which the silicate cloud was always one scale-height thick could account for the change in $J-K$ color from the red L-dwarfs to the blue T-dwarfs. Now using the more physically motivated cloud model of Ackerman \& Marley we can better test this hypothesis.

Figure 3 illustrates the brightness temperature spectra of six brown dwarf models with three different $T_{\text {eff }}$. In the warmest and coolest cases $\left(T_{\text {eff }}=1800\right.$ and $900 \mathrm{~K}$ ) models with and without clouds appear similar. In the warmer case silicate and iron clouds are just forming in the atmosphere and are relatively optically thin, so their influence is slight. In the cooler case as in the righthand image of Uranus in Figure 1, the main cloud deck forms below the visible 

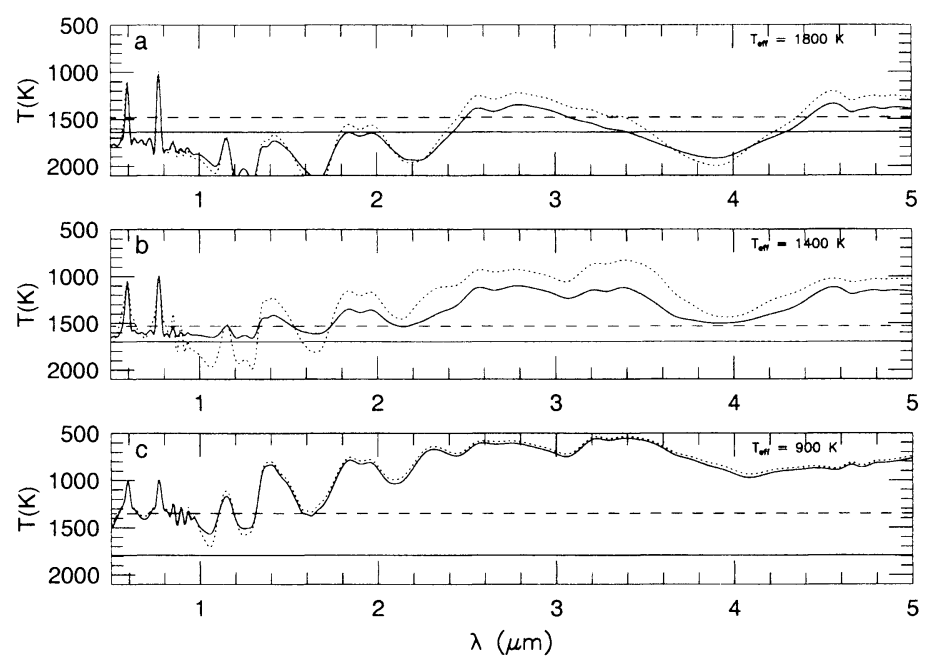

Figure 3. Model brightness temperature spectra from Ackerman \& Marley (2001). Spectra depict approximate depth in the atmosphere at which emission arises. Solid curves depict cloudy models and dotted curves cloud-free models with the same $T_{\text {eff }}$ (all for $g=1000 \mathrm{~m} \mathrm{sec}^{-2} \& f_{\text {rain }}=3$ ). Horizontal dashed and solid lines demark the level at which cloud opacity, integrated from above, reaches 0.1 and the base of the silicate cloud, respectively. In the early$\mathrm{L}$ like model (a) and the T-dwarf like model (c) clouds play a relatively small role as they are either optically thin (a) or form below the level at which most emission arises (c). Only in the late-L case (b) do the optically-thick clouds substantially alter the emitted spectrum and limit the depth from which photons emerge. Cloud base varies with pressure and cloud thickness varies with strength of convection, accounting for the varying cloud base temperature and thickness.

atmosphere. In the intermediate case $\left(T_{\text {eff }}=1400 \mathrm{~K}\right)$ an optically thick cloud forms in the visible atmosphere and substantially alters the emitted spectrum. The atmospheric structure predicted by the Ackerman \& Marley (2001) model for this case is similar to that inferred by Basri et al. (2000) from Cs line shapes in L-dwarf atmospheres. Thus a cooling brown dwarf moves from relatively cloud free conditions to cloudy to clear.

The solid lines in Figure 2 show how the $J-K$ color evolves with $T_{\text {eff }}$. Objects first become red as dust begins to dominate the visible atmosphere, then blue as water and methane begin to absorb strongly in $\mathrm{K}$ band. Models in which the dust does not settle (Chabrier et al. 2000) predict $J-K$ colors much redder than observed. Instead the colors of the L-dwarfs are best fit by models which include some precipitation as parameterized by $f_{\text {rain }}=3$ to 5 . The data clearly require models for objects cooler than the latest L-dwarfs to rapidly change from $J-K \sim 2$ to 0 over a relatively small $T_{\text {eff }}$ range. While models with $f_{\text {rain }}=3$ to 5 do turn blue as the clouds sink below the visible atmosphere (Figure 2), the variation is not rapid enough to satisfy the observational constraints. Ackerman \& Marley suggest that holes in the clouds may begin to dominate the diskaveraged spectra as the clouds are sinking out of sight. Jupiter's 5- $\mu \mathrm{m}$ spectrum is indeed dominated by flux emerging through holes in its clouds. Bailer-Jones 
\& Mundt (2000) find variability in L-dwarf atmospheres that may be related to such horizontal cloud patchiness.

Despite the successes of the Ackerman \& Marley model, clearly much more work needs to be done to understand clouds in the brown dwarfs. Perhaps three dimensional models of convection coupled to radiative transport will be required.

\section{Extrasolar Giant Planets}

The issues of cloud physics considered above of course will also apply to the extrasolar giant planets (Marley 1998; Marley et al. 1999; Seager, Whitney, \& Sasselov 2000; Sudarsky, Burrows, \& Pinto 2000). These papers demonstrate that the reflected spectra of extrasolar giant planets depends sensitively on the cloud particle size and vertical distribution. As already demonstrated by the brown dwarfs in the foregoing section, the emergent thermal flux is similarly affected.

Indeed Sudarsky et al. suggest that a classification scheme based on the presence or absence of specific cloud layers be used to categorize the extrasolar giant planets. Moderate spectral resolution transit observations of close-in EGPs, if the bandpasses are correctly chosen, will certainly provide first-order information on cloud heights and vertical profiles of these atmospheres (Seager \& Sasselov 2000; Hubbard et al. 2001). Coronagraphic multi-wavelength imaging of extrasolar giant planets will provide similar information (see Figure 1).

\section{Conclusion}

It is ironic that although the physics governing the vast bulk of the mass of brown dwarfs and extrasolar planets is very well in hand, the old problem of weather prediction governs the radiative transfer and thus the only remotely sensed quantity. The good news is that there will soon be much more weather to talk about, even if we aren't any farther along in doing anything about it.

Acknowledgments. This work was supported by NASA grant NAG5-8919 and NSF grants AST-9624878 and AST-0086288. The authors benefited from conversations with Dave Stevenson, Sara Seager, Adam Burrows, and Bill Hubbard. Heidi Hammel and Kevin Zahnle offered particularly helpful comments on an earlier draft of this contribution.

\section{References}

Ackerman, A., \& Marley, M. 2001, ApJ, in press

Allard, F., Hauschildt, P. H., Baraffe, I., \& Chabrier, G. 1996, ApJ, 465, L123

Bailer-Jones, C. A. L., \& Mundt, R. 2001, A\&A, in press.

Baines, K. H., \& Hammel, H. B. 1994, Icarus 109, 20

Baines, K., Hammel, H., Rages, K., Romani, P., \& Samuelson, R. 1995, in Neptune (Univ. Ariz. Press), 489

Basri, G., Mohanty, S., Allard, F., Hauschildt, P. H., Delfosse, X., Martín, E. L., Forveille, T., \& Goldman, B. 2000, ApJ, 538, 363

Brooke, T. Y., Knacke, R. F., Encrenaz, T., Drossart, P., Crisp, D., \& Feuchtgruber, H. 1998 , Icarus 136,1

Burrows, A., Marley, M. S., \& Sharp, C. M. 2000, ApJ, 531, 438 
Carlson, B. E., Lacis, A. A., \& Rossow, W. B. 1994 J. Geophys. Res. 99, 114623

Chabrier, G., Baraffe, I., Allard, F., \& Hauschildt, P. 2000, ApJ, 542, 464

Fegley, B. J., \& Lodders, K. 1994, Icarus 110, 117

Griffith, C. A., Yelle, R. V., \& Marley, M. S. 1998, Science 282, 2063

Hubbard, W., Fortney, J., Lunine, J., Burrows, A., Sudarsky, D., \& Pinto, P. 2001, ApJ, submitted

Leggett, S. K., Toomey, D. W., Geballe, T. R., \& Brown, R. H. 1999, ApJ, 517, L139

Liebert, J., Reid, I. N., Burrows, A., Burgasser, A. J., Kirkpatrick, J. D., \& Gizis, J. E. 2000, ApJ, 533, L155

Lunine, J. I., Hubbard, W. B., Burrows, A., Wang, Y. -P., \& Garlow, K. 1989, ApJ, 338,314

Marley, M. S., Saumon, D., Guillot, T., Freedman, R. S., Hubbard, W. B., Burrows, A., \& Lunine, J. I. 1996, Science, 272, 1919

Marley, M. S., Gelino, C., Stephens, D., Lunine, J. I., \& Freedman, R. 1999, ApJ, 513, 879

Marley, M. S. 1998, in Brown dwarfs and extrasolar planets, ASP Conf. Series \#134, eds. R. Rebolo; E. Martin; M. Zapatero Osorio, 383

Marley, M. S. 2000, in From Giant Planets to Cool Stars, ASP Conf. Series \#212, eds. C. Griffith \& M. Marley, 152

Mischna, M. A., Kasting, J. F., Pavlov, A., \& Freedman, R. 2000, Icarus 145, 246

Pollack, J. B., Colburn, D. S., Flasar, F. M., Kahn, R., Carlston, C. E., \& Pidek, D. G. 1979, J. Geophys. Res. 84, 2929

Saumon, D., Geballe, T. R., Leggett, S. K., Marley, M. S., Freedman, R. S., Lodders, K., Fegley, B., \& Sengupta, S. K. 2000, ApJ, 541, 374

Seager, S., \& Sasselov, D. D. 2000, ApJ, 537, 916

Seager, S., Whitney, B. A., \& Sasselov, D. D. 2000, ApJ, 540, 504

Stephens, D., Marley, M., Noll, K., \& Chanover, N. 2001, ApJ, submitted

Sudarsky, D., Burrows, A., \& Pinto, P. 2000, ApJ, 538, 885

Tomasko, M. G., West, R. A., Orton, G. S., \& Teifel, V. G. 1984, in Saturn (Univ, Ariz. Press), 150

Jones, H. R. A., \& Tsuji, T. 1997, ApJ, 480, L39

Tsuji, T., Ohnaka, K., Aoki, W., \& Nakajima, T. 1996, A\&A, 308, L29

Tsuji, T., Ohnaka, K., \& Aoki, W. 1999, ApJ, 520, L119

West, R. A., Strobel, D. F., \& Tomasko, M. G. 1986, Icarus 65, 161 